\title{
Learning from an early pilot of the Dutch Buurtzorg model of district nursing in England
}

Vari M Drennan MBE , Fiona Ross CBE , Melania Calestani , Mary Saunders \& Peter West

District nursing services have struggled in recent years to cope with demand, maintain morale and retain staff. 1,2,3 A Dutch model provided by the social enterprise Buurtzorg has attracted much interest as offering possible solutions. ${ }^{4}$ A central tenet of the Buurtzorg model is 'humanity over bureaucracy' which is enacted in two key strategies. ${ }^{4}$ The first strategy is a patient centred, relationship-based nursing practice ethos with a focus on empowering patients. The second strategy is independent, self-managing teams of nurses, with 40-50 patients, supported by a coach (not a manager) and a small back office for all teams dealing with administrative processes such billing. A number of NHS organisations across the UK are starting to try out this model. We report on some of the learning from a pilot in England.

\section{Neighbourhood Nursing: an adapted pilot of the Buurtzorg model}

A central London NHS trust, facing increasing demands and high vacancy rates, piloted an adapted Buurtzorg model Neighbourhood Nursing (NN) team in order to 'Test and Learn' in late 2016. 'Adapted' in that while the team with their coach followed the Buurtzorg ethos (in nursing practice and self-management), it was still part of a large NHS organisation. A value-based approach was used to recruit the NN team starting with three nurses in November 2016 , increasing to nine by May 2017 before splitting into two teams in June. We undertook an evaluation of the early pilot (January July 2017) : interviewing patients and relatives, GPs, other practitioners and managers , analysing documents and anonymised patient records as well as observing and interviewing neighbourhood nurses and district nurses (DN) . We outline below some of the key findings.

Patients and informal carers were very positive about their experience and the impact on their health and well-being. Those that had received district nursing services before could pinpoint the differences including: continuity in nurse which improved their clinical care and health, the nurse proactively supporting them in addressing their health and well-being problems, direct access to the named nurse and colleagues (rather than an intermediate call centre) and negotiated appointment /telephone contact times. These were issues that patients of the district nursing service interviewed at a similar time raised as ways the service could be improved. The following quotation from a NN team patient illustrates their perceived clinical benefit of the NN team way of working.

"What would happen with the district nurses is you'd get a district nurse who would assess me in a different way; would take a different view. You'd get someone come in - I would say: 'Oh, 
Authors final version post refereeing

they've [the last nurse visiting] stopped doing that [wound management] because [gives reason].' 'Oh, it's all right; this one's [dressing] all right,' [the words of the nurse] and they'd [the different nurse] put it on. So then, the next visit, I was in trouble again [problems with the wound breaking down again] .....So I kept improving and regressing,.... So just one person doing it [wound dressing] different would put me back where I began; which didn't happen with the neighbourhood team at all.” Patient 6

The observation of nurses (neighbourhood and district nurses) confirmed a different approach by the neighbourhood nurses which was much more proactive and responsive to patient needs. It was noted however that at this early stage the NN team had lower numbers of patients than the DN team. Box 1 provides some exemplar statements from the observation of nursing practice.

All DN nurses were pleasant and caring towards patients and competently carried out the task for the patient. However there was a great focus on completing the purpose of the visit e.g. completion of a leg ulcer dressing. The DN nurses appeared to be more task focused in their approach although they did have higher numbers of patients to visit in the same time frame as the NN team. This focus meant that the observer considered that sometimes other potentially important factors were not given attention. .......It was clear to the observer that the NN team had a different approach to care......Many clients gave unsolicited praise for their nurses citing how much they appreciated the continuity of care and the relationship they had with the nurse. Many patients were telephoned before the visit and often a time for the visit was negotiated. For almost every patient, each NN team nurse asked if they could get them anything to eat or drink.....During the visit the NN nurses placed much more focus on the total care of the patients than most of visits observed of the DN nurses. They also spent time offering health promotion and preventative advice.

Box 1 Extracts from the observation of practice report.

The NN team were also making decisions about the way they worked which helped them use their time more efficiently such as streamlining record keeping, making it more meaningful to practice whereas the DN team had multiple and time consuming records. The NN team were also observed to have made a number of decisions about the way they worked which addressed many of the frustrations that the DN nurses described. For example the NN supported each other as a team to ensure they had enough time for each patient, they had flexible rota and shift adjustment (as opposed to weeks in advance as agreed with a senior manager), the NN team made sure they had time back in lieu when they worked extra hours.

The evaluation team examined anonymised patient level records of the NN and DN teams but as the trust at this point was not using a system to classify patients, either by diagnostic group or by severity of condition (s) it was not possible to assess resource input , patient outcomes or costs of either type 
of service. These are key challenges for all community nursing services. The evaluation team were able to identify some of the different activity levels such as much more telephone contact with patients than the DN service. The NN team were also undertaking meal preparation for a small number of patients as an interim measure while social care packages of support were put into place.

The NN nurses were very positive about their experience and emphasised the important role of the coach and having a back office support. They described the satisfaction they received from their work as in this exemplar quote "Providing good holistic care, it fulfils me'. However they also pointed out there were challenges: having to 'unlearn' having managers, having a parent organisation unfamiliar with the concept of self-managing teams as well as being part of a democratically organised team. This was aside from some of their (and the DN teams) frustrations about the lack of mobile technology, particularly having witnessed such in the Dutch Buurtzorg teams. One nurse described the experience of being in the NN team as "It's all good but it is challenging".

Fuller descriptions of all the findings are published elsewhere. ${ }^{5,6}$ This early pilot has led to a second stage with two NN teams and discussion as to how some of the learning can be translated into the ways of working of the wider DN service. There is much that is different between the Dutch health care system and the English NHS, not least that there district nursing services are reimbursed on a tariff basis rather than block contracts. It is evident however that the model offers promise for patients and staff and requires further evaluation over a longer period.

\section{Acknowledgements and disclaimer}

Our thanks and acknowledgement to all the patients, nurses, managers and others who helped us undertake this work.

This was an independent evaluative study was funded by Health Education England (South London), in collaboration with Guy's \& St. Thomas's NHS Foundation Trust. The views expressed are those of the authors and not necessarily the views of the funders or the NHS.

\section{References}

1. Maybin J, Charles A, Honeyman M. Understanding quality in district nursing services. London: Kings Fund. 2016. https://www.kingsfund.org.uk/publications/quality-districtnursing. (Accessed May 2018 2017).

2. NHS Providers Community services: taking centre stage. 2018. http://nhsproviders.org/stateof-the-provider-sector-05-18 (Accessed June 2018)

3. Drennan VM. More care out of hospital? A qualitative exploration of the factors influencing the development of the district nursing workforce in England. J Health Serv Res Policy. 2018 Jan 1:1355819618769082. doi: 10.1177/1355819618769082. 
Authors final version post refereeing

4. De Blok J. Buurtzorg: humanity above bureaucracy in a Teal organization. Presentation at the Chief Nursing Office England Summit Meeting .2015. URL

https://www.slideshare.net/NHSEngland/cnu-summit-2015-buurtzorg-humanity-abovebureaucracy-in-a-teal-organization-jos-de-blok (Accessed June 2018)

5. Drennan VM, Calestani M, Ross F, Saunders M, West P. Tackling the workforce crisis in district nursing: can the Dutch Buurtzorg model offer a solution and a better patient experience? A mixed methods case study. BMJ Open. 2018 Jun 6;8(6):e021931. doi: 10.1136/bmjopen-2018-021931.

6. Drennan, V, Ross, F, Saunders, M and West, P (2017) The Guy's and St Thomas' NHS Foundation Trust Neighbourhood Nursing Team Test and Learn project of an adapted Buurtzorg model : An early view. (Project Report) Faculty of Health, Social Care and Education, Kingston University and St George's University of London. ISBN 9780995741317 http://eprints.kingston.ac.uk/40416/ Accessed last 